\title{
A POSSIBLE MECHANISM OF STELLAR FLARES
}

\author{
YU. V. VANDAKUROV \\ A. F. Ioffe Physical Technical Institute, Academy of Sciences of the U.S.S.R., Leningrad, U.S.S.R.
}

\begin{abstract}
Magnetohydrodynamic considerations on the origin of stellar flares.
\end{abstract}
Instabilities of an emerging magnetic flux tube are considered which might be responsible for the occurrence of stellar flares. During upward motions through a convectively unstable medium, the magnetic field of the tube is assumed to be essentially of a toroidal type. Since convective motions directed mainly along the magnetic field lines are not influenced by the field, the formation of oppositely moving fluid layers in the flux tube (perhaps, in the form of periodic movements) seems to be rather probable. Besides, such fluid motions prove to be necessary to ensure the thermal equilibrium. The point is that the flux tube disturbes the equilibrium distributions in a large area of the convection zone, and the entropy gradient is also among the disturbed quantities (Vandakurov, 1974a). The latter disturbance is not spherically symmetric, generally, and this leads to considerable distortion of the heat flux if the magnetic field is sufficiently strong. Thermal equilibrium becomes possible, when some longitudinal motions whose kinetic energy is close to magnetic energy are present in the tube. The stability of such fluid motions is analyzed in the approximation to a incompressible medium, a stepwise distribution of the velocity, the density and magnetic field being assumed to be continuous with a possible exception for the tube boundary. It proves necessary for the stability that (a) the Alfven velocity is larger than the absolute value of the fluid velocity, and (b) the magnetic pressure is balanced by the difference in temperature outside and inside of the tube. We suppose that, due to the development of instabilities, fluid motions in the tube are formed for which conditions (a) and (b) and the above energy conditions are satisfied (Vandakurov, 1974a, b).

In the course of the upward motion of the flux tube, the magnetic energy is expected to decrease more rapidly than the kinetic energy; hence condition (a) can be violated, and instability of the tangential discontinuity can set in. Interaction of the rising tube with another magnetic field can also be the cause of instability. We suggest this instability of an erupted flux tube to originate stellar flares. Surges and fluid motions are also expected to occur during flux tube eruption. The character of a flare can depend on the rate of tube rise. Note that an acceleration of field rise can take place in a subsurface convective layer with a large value of the density scale height (Vandakurov, 1974b).

\section{References}

Vandakurov, Yu. V.: 1974a, Astron. Zh. 51, 672.

Vandakurov, Yu. V.: 1974b, Solnechnye Dannye (Solar Data), No. 5, 72.

Sherwood and Plaut (eds.), Variable Stars and Stellar Evolution, 91. All Rights Reserved. Copyright (C) 1975 by the IAU. 